\title{
Coach to cope: feasibility of a life coaching program for young adults with cystic fibrosis
}

\author{
This article was published in the following Dove Press journal: \\ Patient Preference and Adherence \\ 21 September 2017 \\ Number of times this article has been viewed
}

\author{
Karin Bæk Knudsen' \\ Tacjana Pressler ${ }^{2}$ \\ Laust Hvas Mortensen ${ }^{3}$ \\ Mary Jarden ${ }^{3,4}$ \\ Kirsten Arntz Boisen ${ }^{5}$ \\ Marianne Skov ${ }^{2}$ \\ Alexandra L Quittner ${ }^{6}$ \\ Terese Lea Katzenstein ${ }^{1,7}$ \\ 'Department of Infectious Diseases, \\ Copenhagen University Hospital, \\ Rigshospitalet, Copenhagen, Denmark; \\ ${ }^{2}$ Cystic Fibrosis Center Copenhagen, \\ Department of Pediatric and \\ Adolescent Medicine, Copenhagen \\ University Hospital, Rigshospitalet, \\ Copenhagen, Denmark; ${ }^{3}$ Department \\ of Public Health, University of \\ Copenhagen, Copenhagen, Denmark; \\ ${ }^{4}$ University Hospital Center for \\ Health Research (UCSF), Copenhagen \\ University Hospital, Rigshospitalet, \\ Copenhagen, Denmark; ${ }^{5}$ Center of \\ Adolescent Medicine, Department of \\ Pediatric and Adolescent Medicine, \\ Copenhagen University Hospital, \\ Rigshospitalet, Copenhagen, Denmark; \\ ${ }^{6}$ Miami Children's Research Institute, \\ Miami, FL, USA; ' ${ }^{7}$ Department of \\ Clinical Medicine, Faculty of Health \\ and Social Sciences, University of \\ Copenhagen, Copenhagen, Denmark
}

Correspondence: Karin Bæk Knudsen Department of Infectious Diseases, Copenhagen University Hospital, Rigshospitalet, Blegdamsvej 9,

DK-2 100 Copenhagen, Denmark

$\mathrm{Tel}+4535453456$

Fax +45 35452975

Email karin.baek.knudsen.0I@regionh.dk
Background: Over the last two decades, lifespan has increased significantly for people living with cystic fibrosis (CF). However, several studies have demonstrated that many young adults with CF report mental health problems and poor adherence to their prescribed treatments, challenging their long-term physical health. Treatment guidelines recommend interventions to improve adherence and self-management. The aim of this study was to test the feasibility of a life coaching intervention for young adults with $\mathrm{CF}$.

Methods: A randomized, controlled feasibility study was conducted at the CF Center at Copenhagen University Hospital, Rigshospitalet. Participants were young adults with CF, aged 18-30 years without severe intellectual impairments. Participants were randomized to either life coaching or standard care. The intervention consisted of up to 10 individual, face-to-face or telephone coaching sessions over a period of 1 year. Primary outcomes were recruitment success, acceptability, adherence to the intervention, and retention rates. Secondary outcome measures included health-related quality of life, adherence to treatment, self-efficacy, pulmonary function, body mass index, and blood glucose values.

Results: Among the 85 eligible patients approached, 40 (47\%) were enrolled and randomized to the intervention or control group; two patients subsequently withdrew consent. Retention rates after 5 and 10 coaching sessions were $67 \%$ and 50\%, respectively. Reasons for stopping the intervention included lack of time, poor health, perceiving coaching as not helpful, lack of motivation, and no need for further coaching. Coaching was primarily face-to-face $(68 \%)$. No significant differences were found between the groups on any of the secondary outcomes. Conclusion: Both telephone and face-to-face coaching were convenient for participants, with $50 \%$ receiving the maximum offered coaching sessions. However, the dropout rate early in the intervention was a concern. In future studies, eligible participants should be screened for their interest and perceived need for support and life coaching before enrollment.

Keywords: life coaching, adherence, depression, quality of life, chronic disease

\section{Background}

Cystic fibrosis (CF) is a genetic, life shortening, multiorgan disease which primarily affects the pulmonary and digestive systems. ${ }^{1}$ Pulmonary exacerbations are the primary cause of increased morbidity and earlier mortality. ${ }^{2}$ However, optimization of medical treatment has improved lifespan, which is now projected to be over 50 years if mortality continues to decrease at the rate observed between 2000 and $2010 .^{3}$ The treatment regimen includes daily preventive home treatments and intensified inpatient or outpatient treatments during pulmonary exacerbations. ${ }^{4}$ Despite inevitable declines in health and the challenges of managing CF on a daily basis, the majority of young adults with CF are either employed or enrolled in a college or university. However, many 
young adults report elevated symptoms of depression and anxiety, ${ }^{5}$ poor adherence to prescribed treatments, ${ }^{6}$ and decrements in health-related quality of life (HRQoL). ${ }^{7}$

Achieving an acceptable balance between taking responsibility for CF care and living a "normal" life, both professionally and socially, is a major challenge. Multiple barriers to adherence to treatment have been identified (ie, time constraints, competing priorities, and accidental and purposeful forgetting). ${ }^{8-10}$ Consensus reports have recommended the development and evaluation of interventions to improve mental health, coping skills, and daily management of the disease. ${ }^{5,11}$

A systematic review of interventions aimed at improving adherence to prescribed CF medications found that even the most effective interventions did not lead to large improvements. ${ }^{12}$ Education and training in self-management (eg, life coaching) may be effective in facilitating patient adherence and management of daily stressors. Modest evidence suggests that self-management education has positive effects on these behavioral changes. ${ }^{13}$ Current evidence for the efficacy of psychological interventions for individuals with $\mathrm{CF}$ is limited. The most convincing results have been found for cognitive-behavioral interventions. ${ }^{14}$ Motivational interviewing (MI), a patient-centered form of counseling which helps patients resolve ambivalence in regard to behavior change, is primarily designed to increase motivation and commitment to change. ${ }^{15} \mathrm{MI}$ is most often used in combination with other programs and moderately efficacious results have been reported. ${ }^{16}$ Coaching interventions overlap with the principles of MI, whereas the focus of MI is primarily on increasing motivation for specific behavior change, coaching is a whole-person model, which includes all life aspects. ${ }^{17} \mathrm{We}$ hypothesized that a coaching intervention would empower young adults with $\mathrm{CF}$ to manage their treatment and daily functioning more effectively, thus improving health outcomes and HRQoL. Few studies have evaluated coaching interventions; thus, the primary aim of this study was to establish the feasibility of a coaching intervention for young adults with CF. Secondary aims included evaluating its effects on health outcomes and HRQoL.

\section{Methods}

\section{Design}

This feasibility study was a prospective, two-arm, randomized controlled trial comparing a life coaching intervention to a control group receiving standard care at a Danish CF Center.

\section{Patients and procedures}

Study participants were recruited at the outpatient CF clinic at the Department of Infectious Diseases at Copenhagen University Hospital, Rigshospitalet, one of two hospitals in Denmark at which CF treatment is centralized. The department treats $\sim 200$ adults with CF. All patients are scheduled to visit the outpatient clinic on a monthly basis. At these visits, the treating physicians (TP and TLK) or investigator and study coach (KBK) informed patients about the study. Patients who did not attend the sessions on a monthly basis received information about the study by letter; if there was no response, phone calls were made to determine the level of interest.

Eligible patients were between 18 and 30 years with a confirmed CF diagnosis by clinical findings, identification of two disease causing CFTR mutations, and a positive sweat chloride test. Exclusion criteria included severe intellectual impairment or insufficient mastery of the Danish language, determined by the inability to complete the questionnaires independently. After written informed consent, baseline data were collected and participants were randomized 1:1 in computer-generated blocks of four, to obtain equal numbers in both arms. We aimed to recruit 40 participants.

This study was conducted in accordance with the Declaration of Helsinki 2004 and all participants signed consent forms. This study was reviewed by the regional ethics committee of the Capital Region of Denmark and deemed exempt from a formal evaluation (H-2-2013-FSP01); it was registered at the Danish Data Protection Agency (j nr 30-0918) and at ClinicalTrials.gov (ID NCT02110914).

\section{Coaching intervention}

All participants were given standard-of-care treatment and were randomized to either life coaching or the control condition. All coaching sessions were delivered by one nurse (KBK) experienced with CF, certified by an Accredited Coaching Training Program, which included 125 student contact hours, 10 hours of mentor coaching, and a performance evaluation. ${ }^{18}$ Participants allocated to coaching (coachees) were offered up to 10 individual coaching sessions. The first and the last coaching sessions were intended to be face-to-face and last up to 90 minutes. Other coaching sessions could be carried out either face-to-face or by telephone, according to participants' preference, and lasted up to 60 minutes. Coaching sessions were scheduled every 1-2 weeks early in the program and every $2-4$ weeks later on. The entire coaching intervention was planned to last 6-9 months. Coaching took place either in a quiet room at 
the hospital outside the Department of Infectious Diseases or at a location close to the hospital.

The intervention was based on a life coaching model that was carried out in successive steps. ${ }^{19,20}$

1. The coach started by building a rapport and encouraging the coachee to tell his/her story.

2. The most important concern for the coachee was identified.

3. Goals were set by the coachee.

4. Barriers for achieving these goals were identified.

5. Collaborative action, consistent with the coachee's values, was planned.

6. Progress was evaluated and new action plans and goals were negotiated.

The coaching intervention included the following elements: client-centeredness, empathy and collaboration, focus on preferences, reflective dialogue, use of positive language, and promotion of capacity and self-determination. ${ }^{21}$ Sessions included different tools; for example, "the wheel of life" 19 on which coachees could rate their level of satisfaction in different life areas. "Time management" 22 was used to help participants structure and prioritize their time. Describing an ideal day or a dream for the future was integrated into the session to identify the participants' values and shortand long-term goals. Finally, participants who reported symptoms of stress were guided to improve sleeping habits, engage in moderate physical exercise, and use mindfulness strategies, according to recommendations from The Danish Health Authority. ${ }^{23}$

\section{Measurements}

\section{Primary study outcome}

The primary outcome for this study was an evaluation of its feasibility. Feasibility measures were described by Bowen et $\mathrm{al}^{24}$ and included the following.

1. Acceptability: How individuals responded to the intervention was assessed by analyzing dropout rates and reasons for dropout.

2. Demand: We assessed interest in this type of intervention by comparing the recruitment rate with the number of eligible participants.

3. Practicality and implementation: Barriers and facilitators for implementation of the coaching intervention were evaluated by documenting the following variables in a logbook for each participant: total number of sessions, their frequency, duration of the coaching sessions, and mode of delivery (telephone or face-to-face). The content of the coaching sessions was confidential, but topics, process, and the use of different tools were also noted in the logbook.

4. Efficacy assessment: Intended effects of the coaching intervention were measured by patient-reported outcomes (PROs) and clinical parameters.

\section{Demographic and disease information}

Baseline demographic data were collected through questionnaires. Disease information was obtained through medical chart review and included CF mutations, body mass index (BMI), lung function, (forced expiratory volume in 1 second $\left.\left[\mathrm{FEV}_{1}\right]\right)$, glycated hemoglobin (HbA1C), chronic lung infection, and CF-related diabetes.

\section{Secondary study outcomes}

The clinical parameters BMI, FEV ${ }_{1}$, and $\mathrm{HbA} 1 \mathrm{C}$, and PRO measures of adherence, depression, HRQoL, and self-efficacy were obtained as secondary outcome measures. The data collection took place at four time points (baseline), midway (after 5 months), post-intervention (after 11 months), and at follow-up (1 year post-intervention). Participants in the intervention group, who stopped after a few coaching sessions, completed the assessment at the same time points as the control group. Adherence was assessed by a validated, 8 -item questionnaire, the Morisky Medication Adherence Scale-8 item (MMAS-8). ${ }^{25-27}$ Adherence to the following treatments was assessed: dornase alfa inhalation, pancreatic enzymes, supplement vitamins (ADEK), inhaled antibiotic therapy, and positive expiratory pressure (PEP) therapy. To assess psychological well-being, two validated depression screening instruments were used: the Major Depression Inventory ${ }^{28}$ and Center for Epidemiologic Studies-Depression Scale. ${ }^{29}$ To assess HRQoL, the Cystic Fibrosis QuestionnaireRevised-Teen/Adult version (CFQ-R) ${ }^{30}$ was completed; it is a validated, disease-specific HRQoL instrument for CF. The CFQ-R assesses demographic information (eg, age, education) and symptoms and functioning across 12 domains: Physical Functioning, Vitality, Emotional Functioning, Eating Disturbances, Treatment Burden, Health Perceptions, Social Functioning, Body Image, Role Functioning, Weight, Respiratory Symptoms, and Digestive Symptoms. Scores are standardized and range from 0 to 100 , with higher scores indicating better HRQoL. The Generalized Self-Efficacy scale, ${ }^{31}$ a 10-item validated scale, measured self-efficacy beliefs.

\section{Statistical analyses}

Baseline comparisons of demographic and disease characteristics were performed by using an independent sample $t$-test for continuous variables and Fishers exact test for 
categorical variables. Descriptive statistics (means and standard deviations [SDs]) were used to describe the sample. Data were analyzed using a two-way repeated measures analysis of variance (ANOVA) with a within-subject factor of time (baseline, midway, post-intervention, and follow-up) and a between-subject factor of group (coaching/control). Missing data were not imputed, and since ANOVA uses listwise deletion for missing data, the sample was reduced to 18 participants (coaching group: $n=7 /$ control group: $n=11$ ). Mauchly's test indicated that the assumption of sphericity had been violated; therefore, degrees of freedom were corrected using Greenhouse-Geisser estimates of sphericity. A $P$-value $<0.05$ was considered statistically significant. Effect size calculations were computed using partial eta squared (partial $\eta^{2}$ ), whereby an effect size of 0.03 is considered small, 0.13 medium, and 0.26 large effects. This feasibility study was not powered to evaluate the efficacy of the intervention. All results should therefore be considered preliminary. Statistical analyses were performed using SPSS version 22 (SPSS Inc, IBM Corporation, Armonk, NY, USA).

\section{Results}

\section{Participant characteristics}

In total, 92 patients aged 18-30 years, with a confirmed diagnosis of $\mathrm{CF}$, were eligible. Three patients were abroad and four patients were excluded due to cognitive impairment. Thus, 85 eligible adults were invited to participate. We continued to enroll individuals until we reached our intended number of 40 participants. This represented $47 \%$ of eligible participants at this adult CF Center. Fifteen young adults declined participation (18\%) and 30 (35\%) never responded. After randomization, two participants withdrew consent; thus, the final sample consisted of 38 participants (intervention, $n=18$ and control, n=20; Figure 1). Demographic and baseline clinical characteristics are shown in Table 1. Most participants were female (71\%), were employed or studying (87\%), and half of the participants $(50 \%)$ had average $\mathrm{FEV}_{1}$ values above $70 \%$ of predicted. The intervention and the control group were comparable, but there were more participants with CF-related diabetes in the intervention group (44\%) versus the control group (20\%) and clinical parameters like $\mathrm{FEV}_{1}$, BMI, and $\mathrm{HbA} 1 \mathrm{C}$ were slightly, but not significantly, worse in the intervention group.

\section{Feasibility}

Feasibility of the study was evaluated using data on acceptability, practicality and implementation, and limited efficacy testing. $^{24}$

\section{Acceptability}

Acceptability was assessed using data on dropout rates and reasons for dropout. Retention rates after 5 and 10 coaching sessions were $67 \%$ and $50 \%$, respectively. Reasons for stopping the intervention included lack of time $(n=1)$, poor health $(n=1)$, coaching not being helpful $(n=2)$, lack of motivation $(n=1)$, no need for further coaching $(n=2)$, and unknown reasons $(n=2)$. Those who stopped because of "no need for further coaching" were not considered dropouts, as they had achieved what they wanted from the coaching intervention in $<10$ sessions. Thus, the dropout rate overall was 39\%.

\section{Practicality and implementation}

In total, 121 coaching sessions were performed. The majority of sessions were face-to-face sessions $(n=82 ; 68 \%)$ and lasted on average 57 minutes (range 20-110). Thirty-nine coaching sessions were carried out by telephone (32\%) and lasted on average 28 minutes (range 5-60). Telephone sessions were most often requested by participants who lived at a long distance from the hospital or who were feeling successful in implementing their action plans; they were primarily interested in having a call to evaluate their progress and, if necessary, adjust their plans. Telephone coaching was also used by participants to save time.

The number of same-day cancelations was 38 (31\%); five $(6 \%)$ were no-shows. Planned telephone coaching calls were not answered 10 times (25\%). Individuals who cancelled or were no-shows for in-person or telephone sessions were offered new appointments. Due to the number of cancellations, it was not possible to adhere to the original schedule of intervals between coaching sessions. The time frame for completion of the coaching program varied; however, those who participated in all 10 sessions completed in 8-15 months. The topics discussed in these sessions also varied across participants. The most frequently chosen topics were 1) work/study-life balance, 2) treatment adherence, 3) stress reduction, 4) time management, 5) establishing priorities, and 6) improving eating or exercise habits. Many participants had difficulty in choosing a topic and setting goals at the first coaching session, but as evidenced by their satisfaction with different areas of life and their personal narrative, they were able to identify topics over time. A few $(n=3)$ either never shared their concerns or stated they did not have any major life challenges.

\section{Secondary outcomes}

Secondary outcomes included patient-reported measures of adherence, depression, HRQoL, and self-efficacy; furthermore, 


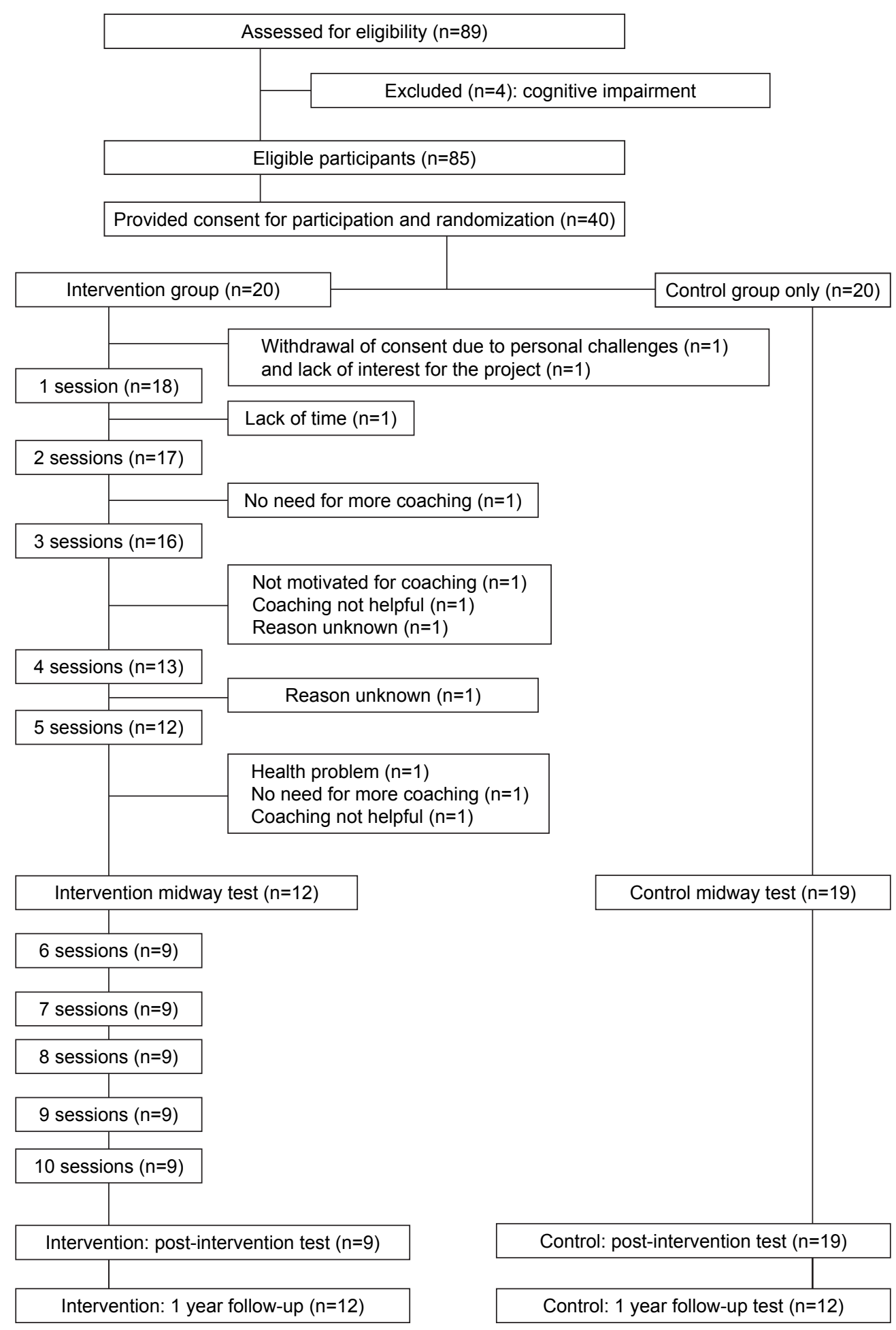

Figure I Participant flow chart.

the clinical parameters $\left(\mathrm{FEV}_{1}\right.$, BMI, or $\left.\mathrm{HbA} 1 \mathrm{C}\right)$ were assessed. The means and SDs of all secondary outcomes for the coaching group and the control group over the four time points are presented in two tables; Table 2 shows outcomes of HRQoL and Table 3 shows all other secondary outcomes.

\section{Adherence}

Adherence was assessed using the MMAS-8 for dornase alfa, pancreatic enzymes, vitamin supplements (ADEK), inhaled antibiotic therapy, and PEP therapy. The scores on the MMAS-8 indicated that many participants (50\%) 
Table I Baseline demographics and clinical characteristics

\begin{tabular}{|c|c|c|c|c|}
\hline & Total & Intervention group & Control group & $P$-value \\
\hline Number & 38 & 18 & 20 & \\
\hline Age, mean (range) & $23.7(18-30)$ & $23.6(18-30)$ & $23.7(18-29)$ & NS \\
\hline Females, n (\%) & $27(7 \mathrm{I})$ & $12(67)$ & $15(75)$ & NS \\
\hline BMI $\left(\mathrm{kg} / \mathrm{m}^{2}\right)$, mean $(\mathrm{SD})$ & $22.1(3.8)$ & $21.7(3.9)$ & $22.5(3.9)$ & NS \\
\hline FEV $\%$ predicted, mean (SD) & $73.4(23)$ & $71.3(24.7)$ & $75.5(21.9)$ & NS \\
\hline $\mathrm{FEV}_{1}(\leq 40 \%), \mathrm{n}(\%)$ & $5(13)$ & $3(17)$ & $2(10)$ & \\
\hline $\mathrm{FEV}_{1}(41 \%-70 \%), \mathrm{n}(\%)$ & $14(37)$ & $7(39)$ & $7(35)$ & \\
\hline $\mathrm{FEV}_{1}(>71 \%), \mathrm{n}(\%)$ & $19(50)$ & $8(44)$ & $11(55)$ & \\
\hline HbAIC\%, mean (SD) & $6.15(1.50)$ & $6.42(1.69)$ & $5.86(1.23)$ & NS \\
\hline CFTR mutation classes I or 2 & $36(95)$ & $18(100)$ & $18(90)$ & NS \\
\hline Chronic pulmonary infection, ${ }^{\mathrm{a}} \mathrm{n}(\%)$ & $21(55)$ & $10(56)$ & II (55) & NS \\
\hline CFRD, n (\%) & $12(32)$ & $8(44)$ & $4(20)^{\prime}$ & NS \\
\hline Single, ${ }^{\mathrm{b}} \mathrm{n}(\%)$ & $19(58)$ & $10(56)$ & $9(45)$ & NS \\
\hline Lower education, $\mathrm{n}(\%)$ & II (29) & $5(28)$ & $6(30)$ & NS \\
\hline High school, n (\%) & $12(32)$ & $5(28)$ & $7(35)$ & NS \\
\hline College/university, n (\%) & $15(39)$ & $8(45)$ & $7(35)$ & NS \\
\hline Employed or studying, $\mathrm{n}(\%)$ & $33(87)$ & $15(83)$ & $18(90)$ & NS \\
\hline Incapacitated to work/study, n (\%) & $3(8)$ & $2(I I)$ & I (5) & NS \\
\hline
\end{tabular}

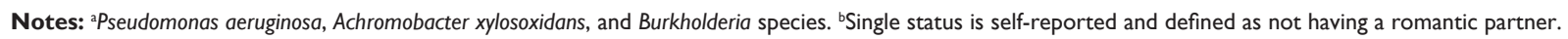

Abbreviations: $\mathrm{FEV}_{\mathrm{l}}$, forced expiratory volume in I second; HbAIC, glycated hemoglobin; CFTR, cystic fibrosis transmembrane regulator protein; CFRD, cystic fibrosisrelated diabetes; NS, nonsignificant; SD, standard deviation; BMI, body mass index.

were poorly adherent to pancreatic enzymes and inhaled antibiotics. However, even worse adherence was reported for PEP treatments and dornase alpha (74\% not adhering) (Table 4). Repeated measures ANOVA showed no significant result for any of the adherence outcomes; a medium effect size was found for dornase alpha over time (partial $\left.\eta^{2}=0.137\right)$ and for PEP over time (partial $\eta^{2}=0.186$ ) (Table 5).

Table 2 Means and standard deviations of health-related quality of life scores from the CFQ-R at baseline, midway, post-intervention, and follow-up

\begin{tabular}{|c|c|c|c|c|c|c|c|c|c|}
\hline \multirow[t]{2}{*}{ Measured outcome } & \multirow[t]{2}{*}{ Treatment } & \multicolumn{2}{|l|}{ Baseline } & \multicolumn{2}{|l|}{$\begin{array}{l}\text { Midway } \\
\text { (5 months) }\end{array}$} & \multicolumn{2}{|c|}{$\begin{array}{l}\text { Post-intervention } \\
\text { (I I months) }\end{array}$} & \multicolumn{2}{|c|}{$\begin{array}{l}\text { Follow-up (I year } \\
\text { post-intervention) }\end{array}$} \\
\hline & & Mean (SD) & $\mathbf{n}$ & Mean (SD) & $\mathbf{n}$ & Mean (SD) & $\mathbf{n}$ & Mean (SD) & $\mathbf{n}$ \\
\hline \multirow[t]{2}{*}{ Physical Functioning } & Coaching & $75(31.6)$ & 18 & $74.4(35.1)$ & 13 & $81.9(23.1)$ & 9 & $68.2(36.8)$ & 12 \\
\hline & Control & 80.2 (19.7) & 20 & $76(26)$ & 17 & $80.3(2 I)$ & 18 & $78.5(30.6)$ & 12 \\
\hline \multirow[t]{2}{*}{ Role Limitations } & Coaching & 82.5 (15.8) & 18 & $80.6(16.8)$ & 12 & 78.1 (19.9) & 8 & 81.1 (13.5) & II \\
\hline & Control & $77.9(20.3)$ & 20 & $73.4(25.3)$ & 17 & $83.8(16.0)$ & 18 & $87.9(14.6)$ & II \\
\hline \multirow[t]{2}{*}{ Vitality } & Coaching & $46.8(21.8)$ & 18 & $5 I .8(2 I)$ & 13 & 49.1 ( 16.4$)$ & 9 & $46.5(22.6)$ & 12 \\
\hline & Control & 48.5 (18.9) & 20 & $50.8(16.0)$ & 17 & $63.9(18.5)$ & 18 & $57.2(2 \mid .2)$ & 12 \\
\hline \multirow[t]{2}{*}{ Emotional Functioning } & Coaching & 67.7 (19.2) & 18 & $69.5(20.7)$ & 13 & $68.1(17.2)$ & 9 & $67.8(21.6)$ & 12 \\
\hline & Control & 7I (2I.2) & 20 & $72.5(17.1)$ & 17 & 76.9 (1 I.3) & 18 & 76.7 (19.2) & 12 \\
\hline \multirow[t]{2}{*}{ Social Functioning } & Coaching & $67.9(22.0)$ & 18 & $72.2(20.9)$ & 13 & $67.9(28.6)$ & 9 & 67.1 (22.2) & 12 \\
\hline & Control & 70.7 ( 18.2$)$ & 20 & 70.7 (16.8) & 17 & $75.9(15.2)$ & 18 & $70.8(24.4)$ & 12 \\
\hline \multirow[t]{2}{*}{ Body Image } & Coaching & $73.5(25.3)$ & 18 & $75.4(18.6)$ & 13 & $72.8(26.1)$ & 9 & $69.4(25.6)$ & 12 \\
\hline & Control & 76.1 (25.8) & 20 & $84.3(20.1)$ & 17 & $79.6(2 I)$ & 18 & 86.1 (15.8) & 12 \\
\hline \multirow[t]{2}{*}{ Eating Disturbance } & Coaching & $89.5(21.7)$ & 18 & $88.9(23.9)$ & 13 & 90.1 (15.2) & 9 & $93.5(11.1)$ & 12 \\
\hline & Control & 89.4 (22.9) & 20 & $88.2(2 \mid .7)$ & 17 & $90.4(15.2)$ & 18 & 86.1 (19.0) & 12 \\
\hline \multirow[t]{2}{*}{ Treatment Burden } & Coaching & $52.5(20.8)$ & 18 & $52.4(24.8)$ & 13 & $51.9(18.4)$ & 9 & $51.9(16.6)$ & 12 \\
\hline & Control & 47.9 (21.9) & 20 & $52.3(22.5)$ & 17 & $56.8(22.2)$ & 18 & $57.4(25.4)$ & 12 \\
\hline \multirow[t]{2}{*}{ Health Perception } & Coaching & $58.6(25.5)$ & 18 & $57.9(29)$ & 13 & $51.9(26.1)$ & 9 & $48.1(26.1)$ & 12 \\
\hline & Control & $58.3(24.9)$ & 20 & $56.9(20)$ & 17 & $60.5(23.9)$ & 18 & $58.3(29.3)$ & 12 \\
\hline \multirow[t]{2}{*}{ Weight Problems } & Coaching & 77.8 (37.9) & 18 & 88.1 (28.I) & 13 & 77.8 (33.3) & 9 & $80.6(26.4)$ & 12 \\
\hline & Control & $83.3(36.7)$ & 20 & $80.4(35.5)$ & 17 & $83.3(30.8)$ & 18 & $80.6(38.8)$ & 12 \\
\hline \multirow[t]{2}{*}{ Respiratory Symptoms } & Coaching & $65.6(19.6)$ & 18 & $61.5(18.7)$ & 13 & $60.5(16.5)$ & 9 & $60.2(23.9)$ & 12 \\
\hline & Control & $67.2(25)$ & 20 & $73.3(21.6)$ & 17 & $70.1(22.6)$ & 18 & $70.8(20.3)$ & 12 \\
\hline \multirow[t]{2}{*}{ Digestive Symptoms } & Coaching & $74.1(25.2)$ & 18 & $65.9(20.7)$ & 13 & $67.9(23.9)$ & 9 & $61.1(23.5)$ & 12 \\
\hline & Control & 7I.I (27.I) & 20 & $81.3(15.0)$ & 16 & 75.7 (18.7) & 16 & 73.7 (25.5) & II \\
\hline
\end{tabular}

Abbreviations: SD, standard deviation; CFQ-R, Cystic Fibrosis Questionnaire revised version. 
Table 3 Means and standard deviations of secondary outcomes at baseline, midway, post-intervention, and follow-up

\begin{tabular}{|c|c|c|c|c|c|c|c|c|c|}
\hline \multirow[t]{2}{*}{ Measured outcome } & \multirow[t]{2}{*}{ Treatment } & \multicolumn{2}{|l|}{ Baseline } & \multicolumn{2}{|l|}{$\begin{array}{l}\text { Midway } \\
\text { (5 months) }\end{array}$} & \multicolumn{2}{|c|}{$\begin{array}{l}\text { Post-intervention } \\
\text { (I I months) }\end{array}$} & \multicolumn{2}{|c|}{$\begin{array}{l}\text { Follow-up ( } 1 \text { year } \\
\text { post-intervention) }\end{array}$} \\
\hline & & Mean (SD) & 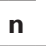 & Mean (SD) & $\bar{n}$ & Mean (SD) & $\mathbf{n}$ & Mean (SD) & $\mathbf{n}$ \\
\hline \multirow[t]{2}{*}{ Dornase alfa ${ }^{a}$} & Coaching & $4.25(2.43)$ & 16 & $4.45(2.44)$ & 13 & $4.67(2.37)$ & 9 & $4.70(1.87)$ & 11 \\
\hline & Control & $3.86(2.73)$ & 18 & $3.72(2.33)$ & 15 & $3.53(2.67)$ & 15 & $2.98(1.77)$ & 10 \\
\hline \multirow[t]{2}{*}{ Pancreatic enzymes ${ }^{a}$} & Coaching & $5.11(2.19)$ & 18 & 4.88 (1.95) & 12 & 5.53 (1.99) & 9 & $5.23(2.15)$ & 12 \\
\hline & Control & $6.08(1.56)$ & 18 & $5.88(1.66)$ & 17 & $6.32(1.49)$ & 17 & $5.39(2.12)$ & II \\
\hline \multirow[t]{2}{*}{ Vitamins supplement ${ }^{\mathrm{a}}$} & Coaching & $5.14(2.56)$ & 18 & $5.13(2.19)$ & 13 & $5.94(2.06)$ & 9 & $5.04(2.30)$ & 12 \\
\hline & Control & $4.93(2.56)$ & 20 & $5.24(2.70)$ & 17 & $5.69(2.5 \mathrm{I})$ & 18 & $5.69(2.5 \mathrm{I})$ & II \\
\hline \multirow[t]{2}{*}{ Inhaled antibiotics ${ }^{a}$} & Coaching & $4.50(2.45)$ & 14 & $4.52(2.45)$ & 13 & $4.97(2.47)$ & 8 & $4.40(2.46)$ & 8 \\
\hline & Control & $5.04(2.54)$ & 18 & $5.03(2.48)$ & 16 & $5.30(2.31)$ & 15 & $4.39(2.32)$ & II \\
\hline \multirow[t]{2}{*}{ PEPa } & Coaching & $3.81(2.66)$ & 16 & $3.92(2.84)$ & 13 & $4.17(2.50)$ & 9 & $3.79(2.55)$ & 12 \\
\hline & Control & $3.62(2.96)$ & 19 & $3.36(2.55)$ & 16 & $3.36(2.76)$ & 16 & $2.78(\mathrm{I} .34)$ & 10 \\
\hline \multirow[t]{2}{*}{ GSE } & Coaching & $32.1(5.7 I)$ & 18 & $33.2(5.27)$ & 13 & $32.9(6.58)$ & 9 & $30.4(6.44)$ & 11 \\
\hline & Control & $32.9(4.12)$ & 20 & $34.1(4.63)$ & 16 & 34.9 (4.39) & 18 & $34.6(4.32)$ & 11 \\
\hline \multirow[t]{2}{*}{ MDI } & Coaching & I 3.6 (7.82) & 18 & I 3.4 (I I.2) & 13 & $11.9(7.03)$ & 9 & $12.9(8.67)$ & 12 \\
\hline & Control & I 3.3 (I0.7) & 20 & $10.9(6.95)$ & 17 & $9.18(6.00)$ & 17 & $10.9(9.76)$ & 12 \\
\hline \multirow[t]{2}{*}{ CES-D } & Coaching & 7.89 (10.8) & 18 & II.3 (II.4) & 13 & 9.71 (7.II) & 7 & $13.3(10.3)$ & 12 \\
\hline & Control & $9.10(9.85)$ & 20 & $12.5(8.94)$ & 16 & 10.1 (8.5I) & 18 & $12.8(10.8)$ & 12 \\
\hline \multirow[t]{2}{*}{$\mathrm{FEV}_{1}$} & Coaching & 71.3 (24.7) & 18 & 74.4 (27.9) & 18 & $69.9(25.3)$ & 18 & $71.0(23.0)$ & 17 \\
\hline & Control & 75.5 (21.9) & 20 & 73.9 (22.5) & 20 & 73.5 (19.2) & 19 & $75.2(23.2)$ & 19 \\
\hline \multirow[t]{2}{*}{ BMI } & Coaching & 21.7 (3.91) & 18 & 22.5 (4.59) & 18 & $22.0(4.23)$ & 18 & $22.2(3.78)$ & 17 \\
\hline & Control & $22.5(3.93)$ & 20 & $22.7(4.06)$ & 20 & 22.1 (3.48) & 19 & $23.2(4.53)$ & 19 \\
\hline \multirow[t]{2}{*}{$\mathrm{HbAlc}$} & Coaching & $6.4 \mathrm{I}(\mathrm{I} .69)$ & 17 & $5.99(2.3 \mathrm{I})$ & 14 & 6.65 (1.76) & 13 & 6.31 (I.53) & 16 \\
\hline & Control & $5.86(1.24)$ & 16 & $6.11(1.13)$ & 14 & $5.96(1.31)$ & 10 & $5.96(0.87)$ & 19 \\
\hline
\end{tabular}

Notes: aScores accessed by MMAS-8. The MMAS (8-item) content, names and trademarks are protected by US copyright and trademark laws. Permission for use of the scale

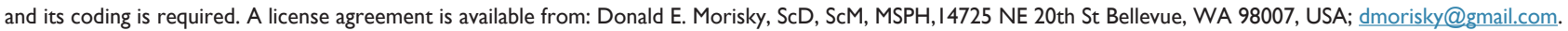
Abbreviations: SD, standard deviation; MMAS-8, Morisky Medication Adherence Scale-8; PEP, positive expiratory pressure; GSE, General Self-Efficacy; MDI, Major Depression Inventory; CES-D, Center for Epidemiologic Studies-Depression Scale; FEV , forced expiratory volume in I second; BMI, body mass index; HbA Ic, glycated hemoglobin.

\section{Depression}

More than one-quarter of patients endorsed elevated symptoms of depression (26.2\%) (Table 4). There were no differences between the groups over time (Table 5).

\section{HRQoL}

At baseline, CFQ-R scores were the lowest (worst) in both groups for Vitality and Treatment Burden and were highest (best) for Eating Disturbances, Weight Problems, and Role

Table 4 Baseline and post-intervention distribution of adherence and depression scores

\begin{tabular}{|c|c|c|c|c|c|c|c|}
\hline & \multicolumn{3}{|c|}{ Baseline } & \multicolumn{2}{|c|}{$\begin{array}{l}\text { Post-intervention } \\
\text { (I I months) }\end{array}$} & \multicolumn{2}{|c|}{$\begin{array}{l}\text { Follow-up (I year } \\
\text { post-intervention) }\end{array}$} \\
\hline & $\begin{array}{l}\text { Total } \\
\text { group } \\
(n=38)\end{array}$ & $\begin{array}{l}\text { Coaching } \\
\text { group } \\
(n=18)\end{array}$ & $\begin{array}{l}\text { Control } \\
\text { group } \\
(n=20)\end{array}$ & $\begin{array}{l}\text { Coaching } \\
\text { group } \\
(n=9)\end{array}$ & $\begin{array}{l}\text { Control } \\
\text { group } \\
(n=18)\end{array}$ & $\begin{array}{l}\text { Coaching } \\
\text { group } \\
(n=12)\end{array}$ & $\begin{array}{l}\text { Control } \\
\text { group } \\
(n=12)\end{array}$ \\
\hline & $\%$ & $\%$ & $\%$ & $\%$ & $\%$ & $\%$ & $\%$ \\
\hline Dornase alfa & $76 \%$ & $75 \%$ & $78 \%$ & $67 \%$ & $80 \%$ & $72 \%$ & $90 \%$ \\
\hline \multicolumn{8}{|l|}{ Low adherence $^{\mathrm{a}}$} \\
\hline Pancreatic enzymes & $50 \%$ & $67 \%$ & $33 \%$ & $67 \%$ & $41 \%$ & $67 \%$ & $55 \%$ \\
\hline \multicolumn{8}{|l|}{ Low adherence ${ }^{a}$} \\
\hline Vitamine supplements & $58 \%$ & $56 \%$ & $60 \%$ & $56 \%$ & $39 \%$ & $67 \%$ & $64 \%$ \\
\hline \multicolumn{8}{|l|}{ Low adherence ${ }^{a}$} \\
\hline Inhaled antibiotics & $56 \%$ & $64 \%$ & $50 \%$ & $63 \%$ & $53 \%$ & $64 \%$ & $64 \%$ \\
\hline \multicolumn{8}{|l|}{ Low adherence ${ }^{a}$} \\
\hline PEP & $74 \%$ & $75 \%$ & $74 \%$ & $78 \%$ & $75 \%$ & $83 \%$ & $100 \%$ \\
\hline \multicolumn{8}{|l|}{ Low adherence $^{a}$} \\
\hline MDI & $15.7 \%$ & $17 \%$ & $15 \%$ & $22 \%$ & 0 & $8.3 \%$ & $8.3 \%$ \\
\hline \multicolumn{8}{|l|}{ Mild depression } \\
\hline MDI & $2.6 \%$ & 0 & $5 \%$ & 0 & $5.6 \%$ & 0 & $17 \%$ \\
\hline \multicolumn{8}{|l|}{ Moderate depression } \\
\hline MDI & $7.9 \%$ & $5.6 \%$ & $10 \%$ & 0 & 0 & $8.3 \%$ & 0 \\
\hline \multicolumn{8}{|l|}{ Severe depression } \\
\hline CES-D depression ${ }^{b}$ & $26 \%$ & $22 \%$ & $30 \%$ & $22 \%$ & $22 \%$ & $25 \%$ & $25 \%$ \\
\hline
\end{tabular}

Notes: aLow adherence: Morisky Medication Adherence Scale-8 item scores <6. 'bepression: CES-D scores > 16.

Abbreviations: PEP, positive expiratory pressure; MDI, Major Depression Inventory; CES-D, Center for Epidemiologic Studies-Depression Scale. 
Table 5 The main effects between the intervention group and the control group over time (baseline, midway, post-intervention, and follow-up) on secondary outcomes

\begin{tabular}{|c|c|c|c|c|c|c|c|}
\hline Measured outcomes & Factor & $P$-value & Partial $\eta^{2}$ & Measured outcomes & Factor & $P$-value & Partial $\eta^{2}$ \\
\hline \multirow[t]{2}{*}{ Physical Functioning ${ }^{c}$} & Time $^{\mathrm{a}}$ & 0.10 & 0.132 & Dornase alpha $^{d}$ & Time & 0.17 & 0.137 \\
\hline & Group $^{\mathrm{b}}$ & 0.73 & 0.008 & & Group & 0.62 & 0.210 \\
\hline \multirow[t]{2}{*}{ Role Limitations $^{c}$} & Time & 0.41 & 0.058 & Pancreatic enzymes $^{d}$ & Time & 0.20 & 0.103 \\
\hline & Group & 0.59 & 0.021 & & Group & 0.68 & 0.012 \\
\hline \multirow[t]{2}{*}{ Vitality $^{c}$} & Time & 0.16 & 0.303 & Vitamins supplement $^{d}$ & Time & 0.55 & 0.046 \\
\hline & Group & 0.31 & 0.064 & & Group & 0.44 & 0.044 \\
\hline \multirow[t]{2}{*}{ Emotional Functioning ${ }^{c}$} & Time & 0.36 & 0.063 & Inhaled antibiotics ${ }^{d}$ & Time & 0.79 & 0.024 \\
\hline & Group & 0.52 & 0.027 & & Group & 0.95 & 0.001 \\
\hline \multirow[t]{2}{*}{ Social Functioning ${ }^{c}$} & Time & 0.67 & 0.138 & PEP $^{d}$ & Time & 0.08 & 0.186 \\
\hline & Group & 0.91 & 0.001 & & Group & 0.32 & 0.076 \\
\hline \multirow[t]{2}{*}{ Body Image ${ }^{c}$} & Time & 0.74 & 0.047 & GSE & Time & 0.31 & 0.094 \\
\hline & Group & 0.26 & 0.078 & & Group & 0.71 & 0.012 \\
\hline \multirow[t]{2}{*}{ Eating Disturbance ${ }^{c}$} & Time & 0.43 & 0.049 & MDI & Time & 0.15 & 0.112 \\
\hline & Group & 0.61 & 0.017 & & Group & 0.35 & 0.059 \\
\hline \multirow[t]{2}{*}{ Treatment Burden ${ }^{c}$} & Time & 0.62 & 0.034 & CES-D & Time & 0.15 & 0.114 \\
\hline & Group & 0.84 & 0.003 & & Group & 0.94 & 0.00 \\
\hline \multirow[t]{2}{*}{ Health Perception ${ }^{c}$} & Time & 0.03 & 0.177 & $\mathrm{FEV}_{1}$ & Time & 0.40 & 0.035 \\
\hline & Group & 0.36 & 0.053 & & Group & 0.91 & 0.001 \\
\hline \multirow[t]{2}{*}{ Weight Problems ${ }^{c}$} & Time & 0.73 & 0.020 & BMI & Time & 0.73 & 0.007 \\
\hline & Group & 0.91 & 0.001 & & Group & 0.62 & 0.012 \\
\hline \multirow[t]{2}{*}{ Respiratory Symptoms ${ }^{c}$} & Time & 0.18 & 0.096 & $\mathrm{HbAIC}$ & Time & 0.17 & 0.226 \\
\hline & Group & 0.07 & 0.197 & & Group & 0.56 & 0.051 \\
\hline \multirow[t]{2}{*}{ Digestive Symptoms ${ }^{c}$} & Time & 0.69 & 0.032 & & & & \\
\hline & Group & 0.68 & 0.013 & & & & \\
\hline
\end{tabular}

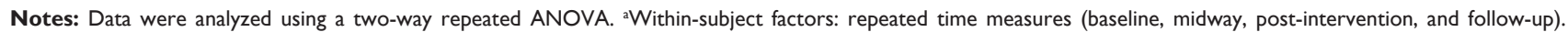
bBetween-subject factors: coaching group $(n=7) /$ control group $(n=11)$. 'Scores assessed by CFQ-R. 'Scores assessed by MMAS-8.

Abbreviations: CFQ-R, Cystic Fibrosis Questionnaire revised version; MMAS-8, Morisky Medication Adherence Scale-8; PEP, positive expiratory pressure; GSE, General

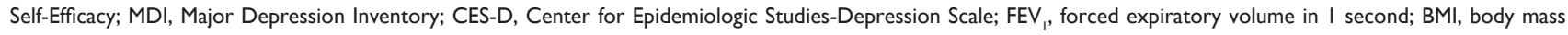
index; HbAlc, glycated hemoglobin; ANOVA, analysis of variance.

Functioning (Table 2). There was no significant difference in any of the CFR-Q outcomes; however, an improvement in the CFQ-R Vitality in the control group gave a large effect size (partial $\eta^{2}=0.303$ ) (Table 5).

\section{Self-efficacy}

The mean self-efficacy score was 33.5 (SD 5.2) of a total score of 40 . There were no differences between the groups over time (Table 5).

No significant improvements in the PRO measures were observed in the coaching group. In addition, no significant improvements were detected in patients' clinical parameters $\left(\mathrm{FEV}_{1}, \mathrm{BMI}\right.$, or HbA1C) (Table 5). Neither within nor between group differences emerged in these outcome measures.

\section{Discussion}

In this study, we evaluated the feasibility of a life coaching intervention for young adults with CF. Using a randomized controlled design, we found that $50 \%$ of participants allocated to the intervention group attended the maximum offered coaching sessions, and both face-to-face and telephone coaching were rated as convenient for participants. This study successfully recruited the desired number of consenting participants, which was set a priori $(n=40)$. This represented $47 \%$ of our clinic sample of eligible young adults with CF. A systematic review of lifestyle interventions reported recruitment rates ranging from $7 \%$ to $28 \% ;{ }^{32}$ thus, recruitment of our target sample, which represented half of our adult clinic population, indicated significant interest in and willingness to participate in a coaching intervention.

Comparing our baseline data with a recent cross-sectional study suggested that our study population was representative of the larger Danish CF adult population in relation to age, disease severity, and educational level. ${ }^{7}$ One key observation was that our participants were primarily female, representing $71 \%$ of our sample. It is possible that a coaching intervention is more appealing to women than men; furthermore, given that the coach was also female, this might also have influenced the gender distribution. Baseline self-efficacy scores 
(mean (SD) 33.5 (5.2)) were higher than the scores among a group of German high school students (mean (SD) 29.6 (4.0)) $(n=3,494) .{ }^{33}$ Self-efficacy is a measurement of an individual's belief in his or her ability to master a given task; thus, the high self-efficacy score could indicate that this group of participants already had good problem solving skills, when they entered the study.

Rates of attrition were $39 \%$ in the coaching group. Attrition rates as high as $18 \%-26 \%$ are not unusual in studies of counseling interventions. ${ }^{34,35}$ Dropout rates in previous studies have been associated with symptoms of depression, e-therapy (mental health services over the Internet), outpatient settings, and interventions that include a large number of sessions. ${ }^{35}$ High dropout rates have also been found in studies targeting behavior change using personal goal setting and identification of barriers. ${ }^{34}$ Many of the challenges associated with high dropout rates also occurred in our intervention. Thus, when designing coaching and other counseling interventions, high rates of attrition should be expected and studies should be powered to assess efficacy taking this dropout rate into account. Strategies that minimize attrition (eg, excluding those that endorse severe depression; offering fewer sessions) should be considered.

We used life coaching as a method for supporting young adults with CF to improve their HRQoL and self-efficacy and to assist them in overcoming barriers to good CF care. All coaching sessions were carried out by a single certified coach, which ensured consistency across participants and sessions. The intervention followed a general model for life coaching; ${ }^{19,20}$ however, since coaching sessions were individualized, the approach was tailored to the specific needs of each participant.

We found that both face-to-face and telephone coaching appealed to participants. Participants preferred face-to-face coaching, but the opportunity to have telephone coaching was useful for those who lived far away or wanted to save time. Telephone coaching was also used to evaluate progress in completing action plans. Face-to-face versus telephone coaching has been evaluated in a few studies with ambiguous results: one study found both methods to be effective, ${ }^{36}$ but another found that face-to-face sessions were superior to telephone coaching. ${ }^{37}$ Our study did not compare the effectiveness of these two methods. Thus, we cannot recommend one method over another. We did, however, find that patients appreciated having both options available.

Participants discussed a variety of topics (eg, work/ study-life balance, treatment adherence, stress reduction, time management, establishing priorities, and improvement of eating or exercise habits). Similar themes have been documented in several studies, indicating that issues related to time management, treatment burden, and adherence are critical challenges for adults with $\mathrm{CF} .{ }^{8-10}$ This also converged with our data on self-reported adherence. Poor adherence was reported by $50 \%-76 \%$ of study participants and this topic was frequently addressed in the coaching sessions. We did not find an association between our intervention and rates of adherence, but the study was not powered to evaluate the efficacy of the intervention.

Stress reduction was also an important topic and this often involved establishing priorities, achieving a better work-life balance, and managing time more effectively. A recent study among young adults with $\mathrm{CF}$ found that $52 \%$ of their study population felt that $\mathrm{CF}$ had a negative impact on education, employment, and other life situations, and the authors state that there is a need for additional support in these issues. ${ }^{38}$ The individual's response to stress can increase vulnerability to depression especially if persistent stress is not resolved through emotion or problem-solved coping or adaption. ${ }^{39}$ It would have been an advantage for our study if we had also included measuring tools to evaluate perceived stress and coping strategies.

The interval between coaching sessions and total length of the coaching program was prolonged due to frequent cancellations. There are no clear recommendations on how frequently coaching sessions should be scheduled, but one of the basic principles of coaching is that the coachees commit themselves to an action plan, with coaching sessions facilitating accountability for these plans. ${ }^{19}$ The risk of relapse during these stages of change may increase if the gap between coaching sessions is too wide.

\section{Limitations}

Although this study is the first to explore the feasibility of a coaching intervention for young adults with $\mathrm{CF}$, it had limitations. First, participants were not initially screened for their need or interest in a coaching intervention. This may have influenced their level of commitment to the intervention, especially for those who did not perceive a need for it. This may have also influenced both cancellations of appointments and dropout rates. Future studies should evaluate interest and level of motivation for participating in a life coaching session. Second, even though this was a feasibility study, which by its nature lacked power to detect any differences, the high dropout rates for the intervention group could have a negative impact of the random assignment; especially concerning are the low response rates for the questionnaires among participants who dropped out. Third, this intervention 
may not be appropriate for those with severe depressive symptoms. Future studies should consider screening out those who express this degree of depressive symptomatology. It is likely that they need a different type of intervention, delivered in a more intensive way. Fourth, to learn more about the individual life stressors and the strategies used to overcome barriers to manage stress in a life with $\mathrm{CF}$, future studies should consider including instruments to measure perceived stress and coping strategies.

\section{Conclusion}

Young adults with CF were willing to participate in a life coaching intervention, and almost half of the participants attended the maximum number of offered coaching sessions. The issues participants chose to work on were related to study/work-life balance, barriers to treatment adherence, and stress. Participants preferred face-to-face coaching, but they also utilized telephone-based counseling when needed. The relatively high dropout rate early in the intervention was a concern. In future studies, participants should be screened for their interest in this type of intervention and those with severely elevated symptoms of depression should be excluded. A study using coaching to reduce stress symptoms among patients with CF should be considered.

\section{Acknowledgments}

The authors thank the participants for their contribution to the study. This was an investigator initiated study. The primary investigator (KBK) is grateful to have received an unconditional grant from Gilead Sciences Inc. and from the Finsen Center, Rigshospitalet. The MMAS (8-item) content, names and trademarks are protected by US copyright and trademark laws. Permission for use of the scale and its coding is required. A license agreement is available from: Donald E. Morisky, ScD, ScM, MSPH,14725 NE 20th St Bellevue, WA 98007, USA; dmorisky@gmail.com.

\section{Disclosure}

The authors report no conflicts of interest in this work.

\section{References}

1. Spoonhower KA, Davis PB. Epidemiology of cystic fibrosis. Clin Chest Med. 2016;37(1):1-8

2. Kerem E, Viviani L, Zolin A, et al. Factors associated with FEV decline in cystic fibrosis: analysis of the ECFS patient registry. Eur Respir J. 2014;43(1):125-133.

3. MacKenzie T, Gifford AH, Sabadosa KA, et al. Longevity of patients with cystic fibrosis in 2000 to 2010 and beyond: survival analysis of the Cystic Fibrosis Foundation patient registry. Ann Intern Med. 2014;161(4): 233-241.
4. O'Sullivan BP, Freedman SD. Cystic fibrosis. Lancet. 2009;373(9678): 1891-1904.

5. Quittner AL, Abbott J, Georgiopoulos AM, et al. International Committee on Mental Health in Cystic Fibrosis: Cystic Fibrosis Foundation and European Cystic Fibrosis Society consensus statements for screening and treating depression and anxiety. Thorax. 2016;71(1):26-34.

6. Quittner AL, Zhang J, Marynchenko M, et al. Pulmonary medication adherence and health-care use in cystic fibrosis. Chest. 2014;146(1): $142-151$.

7. Knudsen KB, Pressler T, Mortensen LH, et al. Associations between adherence, depressive symptoms and health-related quality of life in young adults with cystic fibrosis. Springerplus. 2016;5(1):1216.

8. Sawicki GS, Heller KS, Demars N, Robinson WM. Motivating adherence among adolescents with cystic fibrosis: youth and parent perspectives. Pediatr Pulmonol. 2015;50(2):127-136.

9. George M, Rand-Giovannetti D, Eakin MN, Borrelli B, Zettler M, Riekert KA. Perceptions of barriers and facilitators: self-management decisions by older adolescents and adults with CF. J Cyst Fibros. 2010;9(6):425-432.

10. Bregnballe V, Schiøtz PO, Boisen KA, Pressler T, Thastum M. Barriers to adherence in adolescents and young adults with cystic fibrosis: a questionnaire study in young patients and their parents. Patient Prefer Adherence. 2011;5:507-515.

11. Mogayzel PJ, Naureckas ET, Robinson KA, et al. Cystic fibrosis pulmonary guidelines. Chronic medications for maintenance of lung health. Am J Respir Crit Care Med. 2013;187(7):680-689.

12. Nieuwlaat R, Wilczynski N, Navarro T, et al. Interventions for enhancing medication adherence. Cochrane Database Syst Rev. 2014;11(11): CD000011.

13. Savage E, Beirne PV, Ni Chroinin M, Duff A, Fitzgerald T, Farrell D. Self-management education for cystic fibrosis. Cochrane Database Syst Rev. 2011;7:CD007641.

14. Goldbeck L, Fidika A, Herle M, Quittner AL. Psychological interventions for individuals with cystic fibrosis and their families. Cochrane Database Syst Rev. 2014;6:CD003148.

15. Miller WR, Rollnick S. Motivational Interviewing: Helping People Change. 3rd ed. New York: Guilford Press; 2013.

16. Lundahl B, Moleni T, Burke BL, et al. Motivational interviewing in medical care settings: a systematic review and meta-analysis of randomized controlled trials. Patient Educ Couns. 2013;93(2): $157-168$.

17. Simmons LA, Wolever RQ. Integrative health coaching and motivational interviewing: synergistic approaches to behavior change in healthcare. Glob Adv Health Med. 2013;2(4):28-35.

18. ICF. ACTP Accreditation - Program Accreditation. Available from: https://coachfederation.org/program/landing.cfm?ItemNumber=2151. Accessed August 31, 2017.

19. Whitworth L, Kimsey-House H, Kimsey-House K, Sandahl P. Co-active Coaching: New Skills for Coaching People Toward Success in Work and Life. Palo Alto, CA, USA: Davies-Black Publishing; 2007.

20. Manning S. Coaching. 2nd ed. Oslo, Norway: Aschehoug; 2007.

21. Schwellnus H, King G, Thompson L. Client-centred coaching in the paediatric health professions: a critical scoping review. Disabil Rehabil. 2015;37(15):1305-1315.

22. Covey SR. The seven habits of highly effective people. Natl Med Leg J. 1991;2(2):8

23. Langvarig stress. Aktuel viden og forslag til stress-forebyggelse Rådgivning til almen praksis. [Programme for managing long-term stress]. Center for Forebyggelse. Sundhedsstyrelsen. 2007;25. Danish.

24. Bowen DJ, Kreuter M, Spring B, et al. How we design feasibility studies. Am J Prev Med. 2009;36(5):452-457.

25. Morisky DE, Ang A, Krousel-Wood M, Ward HJ. Predictive validity of a medication adherence measure in an outpatient setting. $J$ Clin Hypertens. 2008;10(5):348-354.

26. Krousel-Wood M, Islam T, Webber LS, Re R, Morisky DE, Muntner P. New medication adherence scale versus pharmacy fill rates in hypertensive seniors. Am J Manag Care. 2009;15(1):59-66. 
27. Morisky DE, Dimatteo MR. Improving the measurement of self-reported medication nonadherence: response to authors. J Clin Epidemiol. 2011;64(3):255-257.

28. Bech P, Timmerby N, Martiny K, Lunde M, Soendergaard S. Psychometric evaluation of the Major Depression Inventory (MDI) as depression severity scale using the LEAD (Longitudinal Expert Assessment of All Data) as index of validity. BMC Psychiatry. 2015;15:190.

29. Radloff LS. The use of the Center for Epidemiologic Studies Depression Scale in adolescents and young adults. J Youth Adolesc. 1991; 20(2):149-166.

30. Quittner AL, Sawicki GS, McMullen A, et al. Erratum to: psychometric evaluation of the cystic fibrosis questionnaire revised in a national, US sample. Qual Life Res. 2012;21(7):1279-1290.

31. Schwarzer R, Jerusalem M. General self-efficacy scale. In: Weinman S, Wright S, Johnston M, editors. Measures in Health Psychology: A User's Portfolio. Causal and Control Beliefs. Windsor, UK: NFER-NELSON; 1995:35-37.

32. Gilinsky AS, Kirk AF, Hughes AR, Lindsay RS. Lifestyle interventions for type 2 diabetes prevention in women with prior gestational diabetes: a systematic review and meta-analysis of behavioural, anthropometric and metabolic outcomes. Prev Med Reports. 2015;2:448-461.
33. Luszczynska A, Gutiérrez-Doña B, Schwarzer R. General self-efficacy in various domains of human functioning: evidence from five countries. Int J Psychol. 2005;40(2):80-89.

34. Crutzen R, Viechtbauer W, Spigt M, Kotz D. Differential attrition in health behaviour change trials: a systematic review and meta-analysis. Psychol Health. 2015;30(1):122-134.

35. Fernandez E, Salem D, Swift JK, Ramtahal N. Meta-analysis of dropout from cognitive behavioral therapy: magnitude, timing, and moderators. J Consult Clin Psychol. 2015;83(6):1108-1122.

36. Opdenacker J, Boen F. Effectiveness of face-to-face versus telephone support in increasing physical activity and mental health among university employees. J Phys Act Health. 2008;5:830-843.

37. Yan T, Wilber KH, Simmons WJ. Motivating high-risk older adults to exercise: does coaching matter? Home Health Care Serv Q. 2011;30(2) 84-95.

38. Askew K, Bamford J, Hudson N, et al. Current characteristics, challenges and coping strategies of young people with cystic fibrosis as they transition to adulthood. Clin Med. 2017;17(2):121-125.

39. Morris MC, Kouros CD, Fox KR, Rao U, Garber J. Interactive models of depression vulnerability: the role of childhood trauma, dysfunctional attitudes, and coping. Br J Clin Psychol. 2014;53:245-263.
Patient Preference and Adherence

\section{Publish your work in this journal}

Patient Preference and Adherence is an international, peer-reviewed, open access journal that focuses on the growing importance of patient preference and adherence throughout the therapeutic continuum. Patient satisfaction, acceptability, quality of life, compliance, persistence and their role in developing new therapeutic modalities and compounds to optimize

\section{Dovepress}

clinical outcomes for existing disease states are major areas of interest for the journal. This journal has been accepted for indexing on PubMed Central. The manuscript management system is completely online and includes a very quick and fair peer-review system, which is all easy to use. Visit http://www. dovepress.com/testimonials.php to read real quotes from published authors. 